\title{
Static Sensitivity of the Aerial Load Transport by Two Rotocopter Unmanned Aerial Vehicles
}

\author{
Jelena ĆOSIĆ LESIČAR, Tomislav RADIŠIĆ, Tino BUCAK, Josip STEPANIĆ
}

\begin{abstract}
Aerial load transport by Unmanned Aerial Vehicles (UAVs) can be performed either using a single UAV or using a group of cooperative UAVs. The latter option is assumed more reliable, yet more demanding regarding the control and required power, than the former one. This article theoretically evaluates power consumption for aerial load transport by the use of two UAVs and compares it with the power consumption in case of a single UAV. In all treated cases, we assume a stationary level flight. For the stated comparison the theoretical model for aerial load transport by two identical UAVs is formulated. Generic flight characteristics and UAVs characteristics are prescribed, and corresponding solutions of the model evaluated. Independent parameters of the model are masses of the UAVs and the load, as well as flight velocity. Variables in the model are vertical and horizontal distances between the UAVs. The emphasis is put on the available instantaneous power and sensitivity to occasional wind gusts. We extract intervals of the model parameters for which each of the two UAVs is less loaded than a UAV which solitary carries a load. The sensitivity to wind gusts is lesser in configurations in which one UAV carries most of the load while the other provides the additionally needed instantaneous power.
\end{abstract}

Keywords: aerial load transport; efficiency; power consumption, static sensitivity; unmanned aerial vehicles

\section{INTRODUCTION}

The aerial load transport by the Unmanned Aerial Vehicles (UAVs) for civilian purposes has been conducted occasionally. The tendency is to augment the number of types of aerial load transport by UAVs and to develop it commercially on a massive scale.

There are two qualitatively different approaches to aerial load transport by UAVs. First approach is to transport a load with a single UAV. That approach utilises a large UAV of a sufficient carrying capacity. This type of UAVs has high requests on multiple control system and other systems important for the flight safety and task conduction reliability. Second approach is to transport a load with a group of UAVs. That approach utilises a cooperatively flying group of UAVs, each of which is uncapable to carry the load solitary.

In literature, cooperative load transport has been dealt with extensively in case of a surface transport (for details see [1] and references therein). On the general level, the cooperative group of UAVs is treated in [2], as a complex system. The authors in [2] research how to make group decisions within a group of UAVs, based on the limited or redundant pieces of information. Here we focus onto more detailed representation of the UAVs, which cannot be directly related to transport by other types of vehicles.

Aerial observation is another application of a group of UAVs. The authors in [3] compared a group of cooperative UAVs with a group of independent UAVs using simulated ground target detection. Specifically, mission completion time and the failure rate were compared. The conclusion of [3] is that a team of cooperative UAVs with individually inferior sensing units outperforms a group of independent UAVs with superior sensing units. Contrary to their case, here we deal with UAVs with a single point (location of the load) emphasised. Group parameters important to us, the power consumption and the sensitivity, differ substantially from those in [3].

In [4] authors divided the set of possible types of UAVs according to their mass and energy. In general, that is global division on hardware level. On the other hand in [5] similar division is conducted on the software level.
The initial flight phase was covered in details, either in case of a single UAV [6] or in case of a swarm of robots [7]. In particular, in [6] the authors analyse lift of the load by a single UAV. That is an important and rather complicated manoeuvre. In [7] the author simulates and experimentally analyses initial gathering of robots into a swarm. Here we suppress the initial and the terminal phases of the flight. It is assumed that most of the flight duration and energy consumption is attributed to the level flight, after the initial and before the terminal phases. We emphasise global characteristics, like energy consumption, contrary to [6] where details of a local control situation are treated or to [7] where substantial variations in geometry of the robots are treated.

In [8] the authors analyse the flight stability and control in case of single UAV load transport. The emphasised element is the influence of the flexible cable between the UAV and the load. Here we also take into account the details of the cable. We concentrate on the stationary level flight (described in the section 2) so a particular cable in our approach is described in a simpler way than in [8]. Yet, in our case there are two cables so overall that is a different source of complexity.

Overall, in the aerial load transport, the use of a single UAV is simpler for flight planning and control. Moreover, that approach is better if tracking of the UAV is available during whole flight. However, the use of a group of UAVs is better in cases when a loss of one or several UAVs has non-negligible probability, in cases in which the flight path passes through the non-characterised or rapidly changing environment, or in cases without flight path tracking. The group, in principle, could be formed using simpler, thus cheaper UAVs. Yet, there are no systematic approaches to characterisation of required power, energy consumption, stability, robustness and the overall capability of the group of UAVs utilised for cooperative aerial load transport.

In this article, we contribute to clarification of the advantages and the deficiencies of the aerial load transport by the group of UAVs if compared to the aerial load transport by a single UAV. We restrict our approach onto the stationary level transport of the load by the two UAVs. 
In particular, we analyse static sensitivity, power demand and energy consumption of the two rotocopter UAVs that simultaneously participate in the aerial load transport during level flight.

The article is organised as follows. In section two, we formulate the model for the load transport by two identical rotocopter UAVs. In section three, we present and discuss its results. In section four, we summarise and conclude the article.

\section{AERIAL LOAD TRANSPORT BY TWO ROTOCOPTER UNMANNED AERIAL VEHICLES MODEL}

We consider the rotocopter UAVs, as that is the prevalent type of UAVs nowadays considered for the aerial load transport. Rotocopters include helicopters, quadrotors and other multicopters such as hexacopters, octocopters, etc. They are suitable for collective load transport because they can hover. With the possibility of hovering, operationally their take-offs and landings are considerably simpler than the take-offs and landings of a group of UAVs with fixed wings. The other type of UAVs that can hover are airships. However, owing to their dimensions and relatively small resistance to horizontal winds their current use is minute which is why we do not consider them further in the text.

UAVs are represented with their aerodynamic characteristic, the coefficient of drag. We cover existing types of UAVs by choosing different values of these coefficients and do not treat any particular UAV separately.

Flight is conducted in the atmosphere with a constant density and other thermodynamics properties. The atmosphere is static most of the time, which is interrupted with the occasional wind gusts parallel to the flight velocity. The wind gusts are taken implicitly in the sense that we find out the geometries formed by relative positions of the UAVs and the load during flight, which are the most robust to occasional external influence, the wind gusts. Realistic flights are conducted within segregated or nonsegregated airspaces [9]. However, here we neglect such aspects of a flight and concentrate on mechanical and energetic considerations.

Each and every complete flight consists of the following three phases: take off, flight in the strict sense and landing. We assume that all these phases include linear trajectories of UAVs and the load. Flight in strict sense is horizontal linear motion, i.e. the level flight. The take-off and landing have either vertical or inclined trajectories. These phases are in the model represented with the corresponding values of vertical and horizontal velocity components. Here we neglect the process of connecting the load to the UAVs. That may be the mooring of cargo while the UAVs are on land, but also the grasping of cargo by the UAVs' manipulators during their hovering [10].

Trajectories for both the UAVs and the load are confined to a single vertical plane during complete flight. In the case considered, symmetry analysis reveals that it is less power-consuming than out-of-plane trajectories.

We consider fixed configuration of the UAVs and the load, so that their relative distances are fixed. Load is connected to the UAVs with two identical non-extensible ropes of lengths $L$ and negligible masses, one rope for each
UAV. All four ends of the ropes are taken to be in the centres of gravities of the corresponding bodies.

Stationary flight configuration is shown in Fig. 1. Corresponding equations are as follows:

$$
\begin{aligned}
& T_{1} \sin \beta_{1}-R_{1 x}=N_{1} \sin \alpha_{1} \\
& T_{1} \cos \beta_{1}-R_{1 y}=N_{1} \cos \alpha_{1}+m_{1} g \\
& T_{1} \sin \beta_{2}-R_{2 x}=-N_{2} \sin \alpha_{2} \\
& T_{2} \cos \beta_{2}-R_{2 y}=N_{2} \cos \alpha_{2}+m_{2} g \\
& N_{1} \sin \alpha_{1}=N_{2} \sin \alpha_{2}+R_{0 x} \\
& N_{1} \cos \alpha_{1}+N_{2} \cos \alpha_{2}=m_{0} g+R_{0 y} \\
& R_{0 x}=\frac{\rho}{2} v_{x}^{2} c_{0 x} S_{0 x}, R_{0 y}=\frac{\rho}{2} v_{y}^{2} c_{0 y} S_{0 y} \\
& R_{1 x}=\frac{\rho}{2} v_{x}^{2} c(\beta) S_{1}, R_{1 y}=\frac{\rho}{2} v_{y}^{2} c(\beta) S_{1} \\
& R_{2 x}=\frac{\rho}{2} v_{x}^{2} c(\beta) S_{2}, R_{2 y}=\frac{\rho}{2} v_{y}^{2} c(\beta) S_{2}
\end{aligned}
$$

Where indices 0,1 and 2 denote the load, the first UAV and the second UAV, respectively. Indices $x$ and $y$ denote projections on the horizontal or vertical axis, respectively. Mass is denoted with $m$ while $g$ denotes the free fall acceleration on the surface of the Earth. Forces treated are weight $G=m \cdot g$, aerodynamic drag $R$, UAV's thrust $T$, and rope tension $N$. Angle between a rope and the vertical axis through load's centre of gravity is denoted as $\alpha$. Angle which an UAV's thrust vector forms with vertical is denoted as $\beta$, and it has the meaning of pitch angle. Angle, which an UAV's aerodynamic drag force vector forms with vertical, is denoted as $\gamma . S$ is the area of the vertical projection of an object and $\rho$ is local air density. Drag coefficients $c$ for UAVs depend on the direction of thrust vector $\beta$. In Fig. $1, r$ denotes horizontal projection of a distance between the load and an UAV, while $L$ is a rope length. Finally, $h$ is relative height among UAVs and $d$ the horizontal projection of their relative distance. Along with masses, also the aerodynamic drags of the ropes are neglected. (1)-(9):

The following conditions accompany the set of Eqs.

$N_{1,2}>0, \alpha_{1,2}>0$

Left condition in (10) assures that both UAVs carry the load, while right condition excludes configurations, which are symmetrical for interchange of indices 1 and 2. For convenience, we consider the geometry to be known if $d$ and $h$ are determined.

Solutions to the formulated set of equations are as follows:

$$
\begin{aligned}
& T_{1}=\frac{R_{1 x} \cos \alpha_{1}-\left(R_{1 y}+m_{1} g\right) \sin \alpha_{1}}{\sin \left(\alpha_{1}-\beta_{1}\right)} \\
& T_{2}=\frac{\left(R_{2 y}+m_{2} g\right) \sin \alpha_{2}+R_{2 x} \cos \alpha_{2}}{\sin \left(\alpha_{2}+\beta_{2}\right)}
\end{aligned}
$$




$$
\begin{aligned}
& \tan \beta_{1}=\frac{R_{1 x}+\sin \alpha_{1} \frac{\left(m_{0} g+R_{0 y}\right) \sin \alpha_{2}+R_{0 x} \cos \alpha_{2}}{\sin \left(\alpha_{1}+\alpha_{2}\right)}}{R_{1 y}+m_{1} g+\sin \alpha_{1} \frac{\left(m_{0} g+R_{0 y}\right) \sin \alpha_{2}+R_{0 x} \cos \alpha_{2}}{\sin \left(\alpha_{1}+\alpha_{2}\right)}} \\
& \tan \beta_{2}=\frac{R_{2 x}-\sin \alpha_{2} \frac{\left(m_{0} g+R_{0 y}\right) \sin \alpha_{1}-R_{0 x} \cos \alpha_{1}}{\sin \left(\alpha_{1}+\alpha_{2}\right)}}{R_{2 y}+m_{2} g+\sin \alpha_{2} \frac{\left(m_{0} g+R_{0 y}\right) \sin \alpha_{1}-R_{0 x} \cos \alpha_{1}}{\sin \left(\alpha_{1}+\alpha_{2}\right)}}
\end{aligned}
$$

$$
\begin{aligned}
& \alpha_{1}=\cos ^{-1}\left\{\frac{1}{2 L}\left[-h+d \sqrt{\frac{4 L^{2}-h^{2}-d^{2}}{h^{2}+d^{2}}}\right]\right\} \\
& \alpha_{2}=\cos ^{-1}\left\{\frac{1}{2 L}\left[h+d \sqrt{\frac{4 L^{2}-h^{2}-d^{2}}{h^{2}+d^{2}}}\right]\right\} .
\end{aligned}
$$

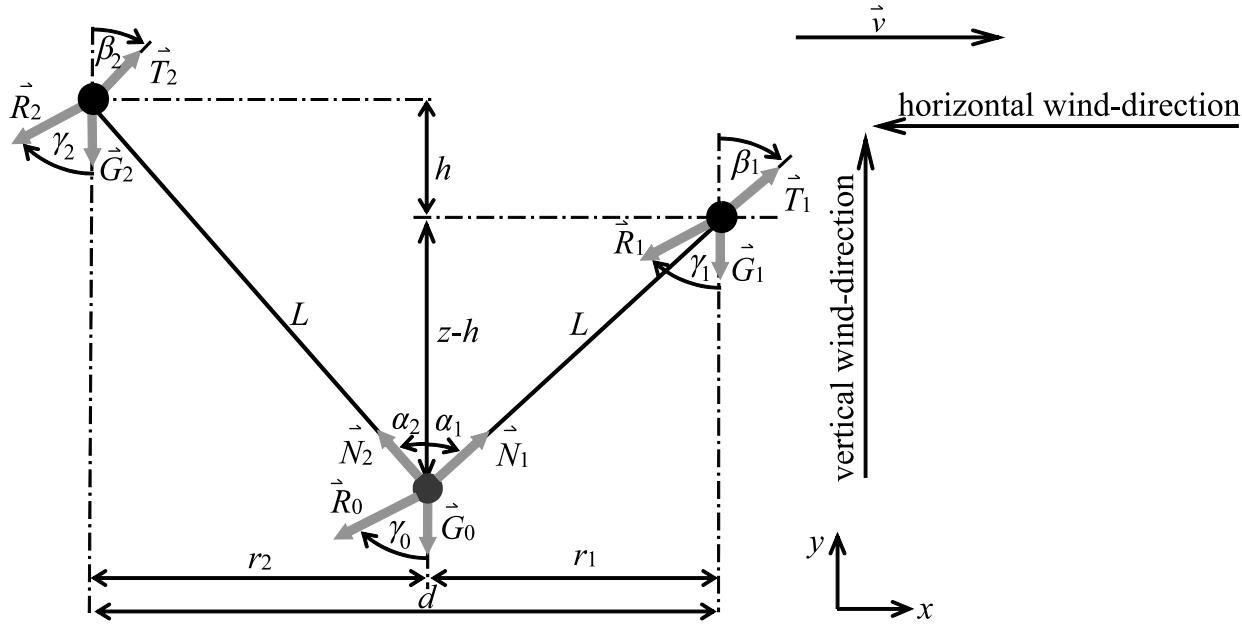

Figure 1 Configuration of the UAVs and the load in the vertical plane containing velocities. Both the UAVs and the load are represented with the circles. Flight direction is to the right. Assumed directions of the wind gust are horizontal (parallel to $x$ ) and vertical (parallel to $y$ ).

Without loss of generality, we take $v_{x} \geq 0$. Vertical flight is taken into account with the condition $v_{x}=0$ and horizontal flight with $v_{y}=0$. Consequently, for both the UAVs and the load during vertical flight one has $R_{x}=0$ and during horizontal flight $R_{y}=0$.

There is a variety of UAVs and loads regarding their shapes. In all cases, their drag coefficients enter the model entirely through aerodynamic drag forces $R$. Therefore, further in the text we do not consider explicitly drag coefficients. Instead, we cover possible realisations of UAVs and the load with different values of the corresponding aerodynamic drag forces.

Total thrusts realised by an UAV, $T_{1,2}$, are in case of a multi-rotor rotocopter combination of thrusts realised by single rotors. In case of $n$ identical, equally loaded rotors forming a rotocopter UAV we utilise the following expression:

$P=n P_{\mathrm{r}}$

in which $P$ is the total power realised by a rotocopter UAV and $P_{\mathrm{r}}$ is the power realised by any of its rotors. In (16) we take that all rotors are equally loaded, and we neglect the interference effects. We do not consider in details the rotation of UAVs around horizontal axis as it is a transient motion. Power realised at a particular rotor is related to the thrust of that rotor, $T_{\mathrm{r}}$, with the following expression:

$P_{\mathrm{r}}=\sqrt{\frac{T_{\mathrm{r}}^{3}}{2 \rho A_{\mathrm{r}}}}$ in which $A_{\mathrm{r}}$ denotes the area spanned by a single rotor. Since the total thrust of a UAV satisfies

$T=n T_{\mathrm{r}}$

Combining (16)-(18) one obtains

$P_{1,2}=\sqrt{\frac{1}{2 \rho A}} \cdot T_{1,2}^{3 / 2}$

in which we approximated the area of the rotocopter with the area spanned by all of its rotors: $A=n A_{\mathrm{r}}$. The instantaneous power generated by both UAVs equals:

$$
P\left(m_{0}, m_{1}, m_{2}, v, d, h\right)=P_{1}+P_{2}=\sqrt{\frac{1}{2 \rho A}} \cdot\left(T_{1}^{3 / 2}+T_{2}^{3 / 2}\right)
$$

Where its dependency on other quantities is explicitly stated. Among possible values of $d$ and $h$, of particular importance are the values, denoted as $d_{P}$ and $h_{P}$, for which (20) achieves its minimal value, denoted as $P_{\min }$ :

$P_{\min }\left(m_{0}, m_{1}, m_{2}, v\right)=P\left(m_{0}, m_{1}, m_{2}, v, d_{P}, h_{P}\right)=$

$=\min _{d, h} P\left(m_{0}, m_{1}, m_{2}, v, d, h\right)$

Energy $E$, consumed during traversing the fixed distance $D$ with constant powers generated on $\mathrm{UAVs}$, is

$$
E\left(m_{0}, m_{1}, m_{2}, v, d, h\right)=\frac{D\left(m_{0}, m_{1}, m_{2}, v, d, h\right)}{v}
$$


Among energies consumed for different geometries, of particular importance is the minimal consumed energy $E_{\min }$, achieved for a particular geometry fixed by $d_{E}$ and $h_{E}$, and for a particular speed $v_{E}$ :

$$
\begin{aligned}
& E_{\min }=E\left(m_{0}, m_{1}, m_{2}, v_{E}, d_{E}, h_{E}\right) \approx \\
& \approx \min _{v, d, h} \frac{P\left(m_{0}, m_{1}, m_{2}, v, d, h\right)}{v}
\end{aligned}
$$

where we suppressed the distance $D$ from the last expression since $E_{\min }$ is proportional to $D$. Generally, by putting different number of batteries into the UAVs one can modify their masses. We furthermore assume that a fixed battery can provide the UAV with sufficient energy within a range of needed energies. Therefore, we exclude the possibility of variable masses of UAVs' for different achievable geometries and related energies.

Instead of taking explicitly into account speed $v$, we express it as $\sqrt{R_{x}}$ with additional assumption that the drag coefficients in (7)-(9) do not change significantly. Following that, a change in the values of $R_{x}$ incorporates both the changes in shapes and the changes in the overall speed in the horizontal direction.
In order to obtain the quantitative parameters describing usefulness of the system of UAVs from the point of view of energy efficiency, as a referent case we consider the aerial load transport by a single UAV, Fig. 2 . After straightforward derivation, the UAV's thrust $\tau$ in that case equals

$$
\begin{aligned}
& \tau=\left[R_{0}^{2}+R_{1}^{2}+2\left(R_{1 x} R_{0 x}+R_{1 y} R_{0 y}\right)+\right. \\
& \left.+2\left(G_{1}+G_{0}\right)\left(R_{1 y}+R_{0 y}\right)+\left(G_{1}+G_{0}\right)^{2}\right]^{1 / 2}
\end{aligned}
$$

Corresponding instantaneous power $\pi$ and minimal energy $\varepsilon$ are given as follows:

$$
\begin{aligned}
& \pi=\pi\left(m_{0}, v\right)=\sqrt{\frac{\tau^{3}}{2 \rho A}} \\
& \varepsilon=\varepsilon\left(m_{0}, v_{\varepsilon}\right)=\min _{v} \frac{\pi\left(m_{0}, v\right)}{v}
\end{aligned}
$$

with $v_{\varepsilon}$ being the speed of flight for which the minimal energy is consumed. Note that values of equally denoted quantities in Figs. 1 and 2 generally differ mutually.

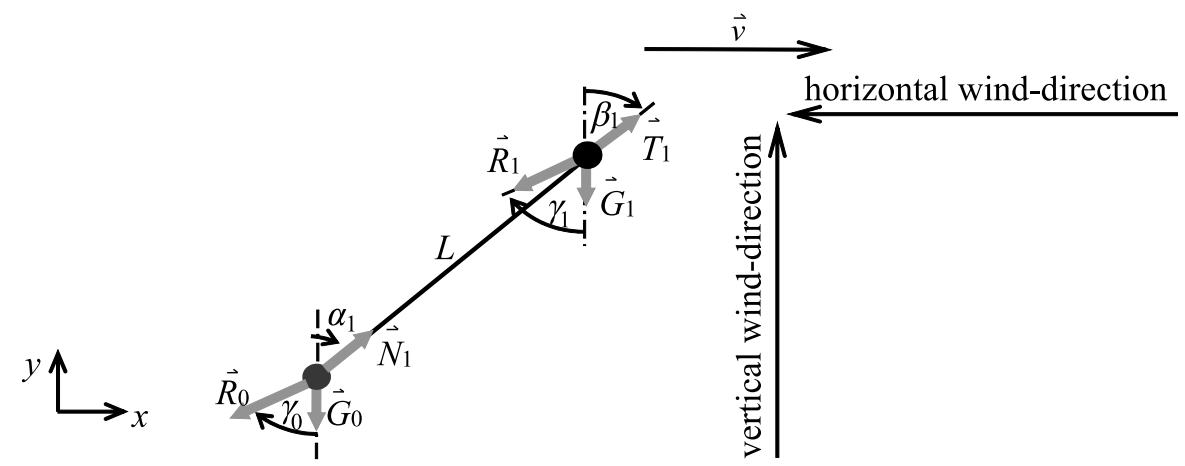

Figure 2 Configuration of the single UAV and the load in the vertical plane containing velocities. Both the UAV and the load are represented with the circles. Assumed directions of the wind gust are horizontal (parallel to $x$ ) and vertical (parallel to $y$ ).

Power factor $f_{P}$ and energy factor $f_{E}$ compare powers and energies, respectively, which are generated and consumed in the aerial load transport by two UAVs, to the corresponding quantity for a solitary UAV:

$f_{P}=\frac{P_{\min }}{\pi}, f_{E}=\frac{E_{\min }}{\varepsilon}$

Finally, a quantity describing the overall behaviour of the system is its sensitivity to the occasional wind gusts. Here we reduce the changes of wind to the changes of wind velocity in the plane containing the UAVs and the load. Thus wind flows are horizontal (front wind and back wind) or vertical (up-wash or down-wash). The assumed response of the system to changes in any of these flows is observed firstly as the change in geometrical parameter defined in direction of the external change. Second observation is as the change in generated power which is needed to preserve the final geometry. If the geometry is preserved, one can consider the system to have a sufficient stability. However, that preservation requires a change in generated power. For assumed infinitesimal changes of the initial geometry we calculate the differences in the instantaneous power (20), generated in the initial and the infinitesimally changed geometry. The larger that difference the more sensitive is the system to a wind gust.

We introduce static sensitivity, the quantitative measure of system's response to occasional wind gusts, as follows:

$$
\sigma(d, h)=\sqrt{\left(\frac{\partial f_{P}}{\partial d}\right)^{2}+\left(\frac{\partial f_{P}}{\partial h}\right)^{2}}
$$

Before proceeding let us note that during flight, from the point of view of the UAVs and the load, there is a constant wind of the velocity $\vec{v}$. The relative wind speed is assumed variable because of the wind gusts Static sensitivity $\sigma$ is a quantity evaluated based on the static configurations, yet it provides one with an insight into the dynamics of a system. Too large sensitivity implies the unstable configuration. On the contrary, small sensitivity implies statically stable configuration as the needed change in instantaneous power is relatively small, thus assumed achievable by UAVs' motors. 


\section{$3 \quad$ RESULTS OF SIMULATED MODEL}

We show results for $m_{1} / m_{0}=m_{2} / m_{0}$ and equal to $0.1,1$ and 10. Trough different magnitudes of its aerodynamic drag different shapes of the load are taken. In simulations aerodynamic drags $R_{0 \mathrm{x}} / \tau$ and $R_{0 \mathrm{y}} / \tau$ are taken to be equal to 1 or 10 . We take $n=4$, which is valid for quadrotor UAVs. Differences for other rotocopter UAVs are merely quantitative, not substantial.

Dependence of thrusts $T_{1,2}$ on $d$ with fixed $h$ is shown in left graphs in Figs. 3-5. These graphs reveal three typical regions. First region has relatively small $d$ and for that region we considered $d / L<<1$. Second region encompasses moderate $d$. For that region we considered $d / L \approx 1$. Last region is region of relatively large $d$ and $d / L \approx 2$.

For relatively small $d / L$ and relatively small load drag force $R_{0 x}$ (Fig. 3) prevalently one UAV carries the load while the other contributes negligibly to the load transport.
Here it is the back UAV (denoted as UAV 2) that carries most of the load while the UAV in front (denoted as UAV 1) from the point of view of flight direction, carries prevalently itself. That is seen in the graph for rope tensions $N_{1,2}$ (left graph in Fig. 6) since for $d / L$ tension of the rope connecting the load with the front UAV is negligible. For relatively large $d / L$, still in case of relatively small load drag force $R_{0 x}$ (Fig. 3) both thrusts diverge. Corresponding graph of rope tensions (left graph in Fig. 6) reveals that the tensions $N_{1,2}$ are considerably larger than the total load weight $G_{0}$. In the region of moderate values of $d / L$ there is gradual change of thrusts with $d$. Depending on the relative weights of UAVs $m_{1,2} / m_{0}$ and on the load drag $R_{0 x}$, UAVs may switch their prevalent contribution to load carrying, as marked by line crossings in left graphs in Figs. 3 and 5, but not in Figure 4. In case of relatively large load drag force $R_{0 x}$, UAVs share their contribution to load carrying for relatively small and moderate $d / L$.
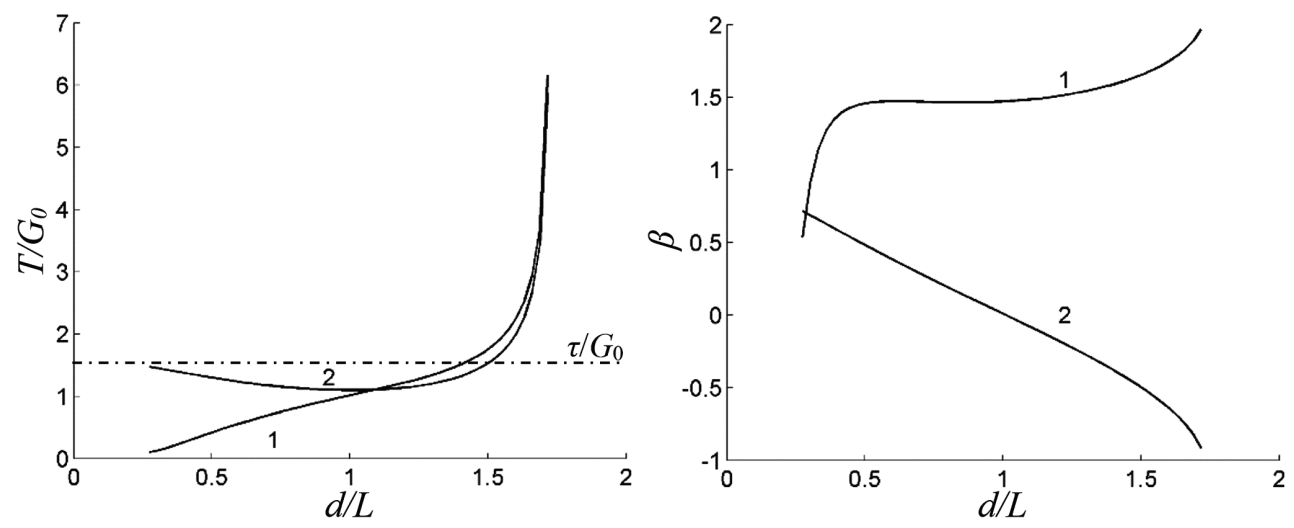

Figure 3 Thrusts (left) and pitch angles (right) of UAVs shown as functions of $d$ during level flight for $h / L=1, m_{1,2} / m_{0}=0.1, R_{1,2}=0$ and $R_{0 x} / G_{0}=1$
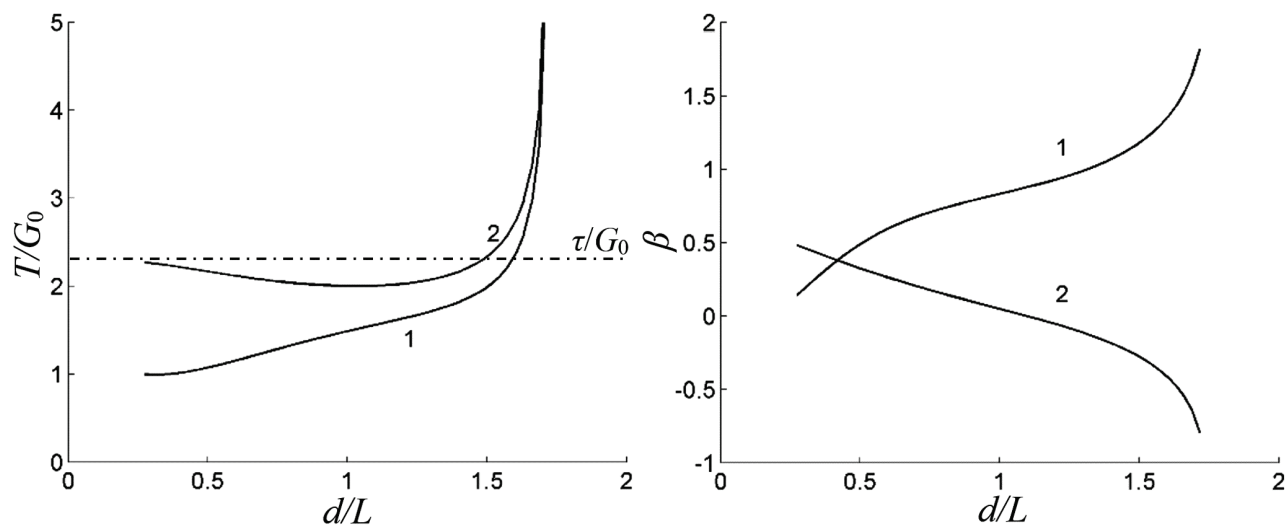

Figure 4 Thrusts (left) and pitch angles (right) of UAVs shown as functions of d during level flight for $h / L=1, m_{1,2} / m_{0}=1, R_{1,2}=0$ and $R_{0 x} / G_{0}=1$
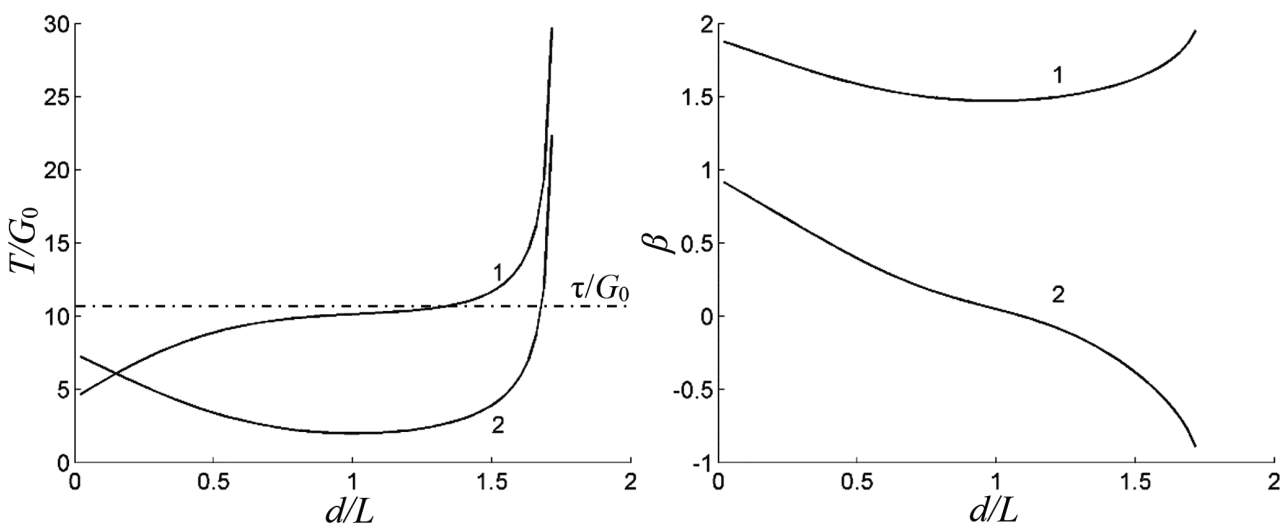

Figure 5 Thrusts (left) and pitch angles (right) of UAVs shown as functions of $d$ during level flight for $h / L=1, m_{1,2} / m_{0}=1, R_{1,2}=0$ and $R_{0 x} / G_{0}=10$ 
For most of the geometries represented in Figs. 3-5, one has $T_{1,2}<\tau$, so the thrusts of each of the two UAVs is smaller than the thrust of a UAV in a solitary load transport. That is a manifestation of the cooperative load transport. Fig. 7 shows that the sum of amounts of thrusts is almost constant for most of the $d / L$.

Independently to the relative amount of the load drag force $R_{0 x} / G_{0}$, thrusts $T_{1,2}$ diverge for large separation of UAVs, i.e. for large $d / L$. That divergence is a sign of intense competition of the UAVs. For large $d / L$ the UAVs prevalently compete. Each UAV pulls on its side, so that only a minute amount of their total thrusts contributes to the load carrying. Competitive and cooperative characters of UAVs functioning are related to the UAVs configuration in such a way that for sufficiently large $d / L$ the competition prevails.

The pitch angles of UAVs, $\beta_{1,2}$, that correspond to their thrusts are shown in right graphs in Figs. 3-5. In the case of relatively small load drag force (right graphs in Figs. 3 and 4), the pitch angle of the front UAV lies between the $0^{\circ}$ and $90^{\circ}$ for small and moderate values of horizontal separation $d$. The initial pitch angle of the front UAV, as it negligibly contributes to the load carrying for relatively small values of $d$ in the case of relatively small load drag force $R_{0 x}$, depends solely on its weight and is smaller for its larger mass. In other words, then the front UAV's thrust points closer to vertical axis in case of its larger mass. In the region of moderate values of $d / L$ the pitch angle of the front UAV, $\beta_{1}$, gradually rises and for relatively large $d / L$ exceeds $90^{\circ}$ hence that UAV's thrust vector points below local horizontal axis. In case of relatively large load drag force $R_{0 x}$ the pitch angle $\beta_{1}$ is larger than $90^{\circ}$ in the whole region of achievable separations $d$. Contrary to that, the pitch angle of the back UAV, $\beta_{2}$, qualitatively does not depend on the load drag force $R_{0 x}$, and shows typical behaviour for all ratios of $m_{1,2} / m_{0}$ as a function of horizontal separation $d$ : for relatively small $d / L$ it is positive and smaller than $90^{\circ}$ so its thrust vector points upwards, yet has relatively large angular separation from the vertical axis. For $d / L \approx 1$ the pitch angle of the back $\mathrm{UAV}, \beta_{2}$, equals $0^{\circ}$ and for larger $d / L$ it becomes negative. The back UAV's thrust vector, thus points upwards but against the flight direction. That is another manifestation of the enlarged competition between the UAVs.

Some combinations of $d$ and $h$ are not allowed. Some parts of the square $0 \leq d, h \leq 2 L$ are excluded because the ropes are non-extensible. Another part is excluded because the UAVs have finite dimensions and wake area, so they cannot come too close to each other. Because of that the region in the vicinity of $d \approx h \approx 0$ is excluded. If, only for theoretical purposes, one analyses the parametric region $d / L, h / L \approx 0$ one obtains that total thrust resembles $\tau$, which is aligned with the fact that in these cases UAVs have almost identical thrust vectors, with the amount equal to half of the thrust that the solitary UAV needs to transport the same load.
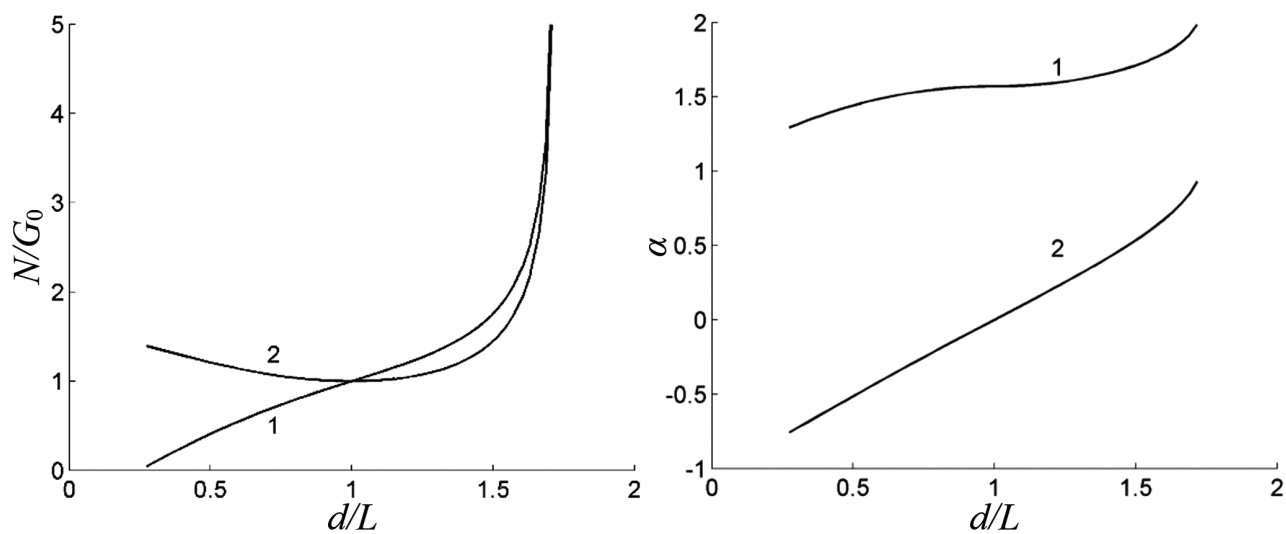

Figure 6 Rope tensions (left) and angles that ropes form with the vertical (right) during level flight, shown as functions of $d$ for $h / L=1, m_{1,2} / m_{0}=1, R_{1,2}=0$ and $R_{0 x} / G_{0}=1$
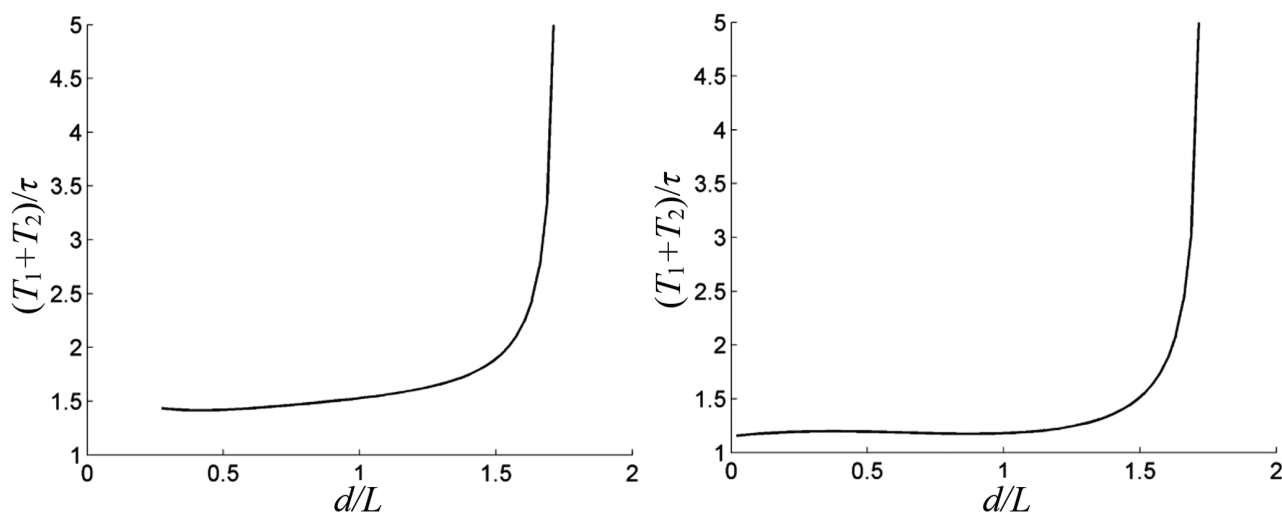

Figure 7 Relative sum of amounts of UAVs' thrusts as a function of $d$ for $h / L=1, m_{1,2} / m_{0}=1$. Left (right) graph is for $R_{0 x} / G_{0}=1\left(R_{0 x} / G_{0}=10\right)$

Sensitivity, shown in Fig. 8, depends non-trivially on geometrical parameters $d$ and $h$. It diverges in the vicinity of $d, h \approx 0$ because the fixed amount of change of either $d$ or $h$ represents large relative change. Furthermore, sensitivity diverges for larger distance $\left(d^{2}+h^{2}\right)^{1 / 2}$ between the UAVs because in that region the competition between 
the UAVs is considerable (projected on a line for fixed $h$ that is the interval of large $d$ in graphs on the left in Figs. 4, 5 and 7). Hence relatively small change in geometry considerably changes required thrusts and corresponding generated powers. Between these two regions of diverging sensitivity is a saddle-like region containing the minimal sensitivity. In realistic conditions of turbulent atmosphere, that region encompasses the useful configurations. Indeed, minimal sensitivity means that minimal additional power needs to be generated in order to preserve the configuration after its sudden yet relatively small change. Precise amount of sensitivity in that useful region depends on the overall speed (expressed in terms of drag force in the level flight) and is larger for faster flights.

Let us further analyse the configurations bringing about the global minima of sensitivities. For that purpose, we focus onto the corresponding thrusts and analyse trends in their change because of the change of $d$ (using left graphs in Figs. 4 and 8). It is seen that each of the thrusts changes minimally with the change in $d$ for fixed $h=1$, thus bringing about the minimal overall change in total instantaneous power for variable $d$. Cases with fixed $d$ and variable $h$ reveal the similar dependence, which is why both of the partial derivatives in (28), hence also the complete sensitivity, are minimal.
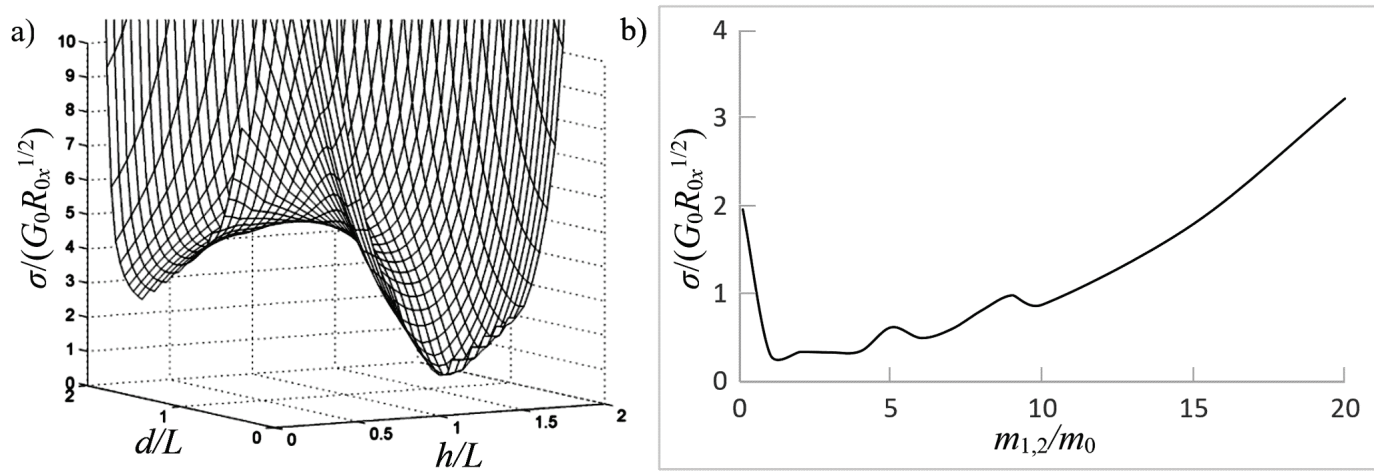

Figure 8 a) Sensitivity $\sigma$ from (28) for $m_{1,2} / m_{0}=1$, b) minima of sensitivity shown as function of $m_{1,2} / m_{0}$. For both graphs $R_{0 x} / G_{0}=1$

Right graph in Fig. 8 shows further details regarding the minimal sensitivity. It shows the dependence of minimal sensitivity on ratio $m_{1,2} / m_{0}$. The sensitivity achieves its global minimum for relatively small values of $m_{1,2} / m_{0}$, equal or slightly greater than 1 . For rising $m_{1,2} / m_{0}$, still in the vicinity of $m_{1,2} / m_{0} \approx 1$, one can observe the change in geometry for which minimal sensitivity is obtained (right graph in Fig. 8). In that region, height difference $h$ between the UAVs is relatively constant while lateral separation $d$ becomes smaller. Both UAVs lie in front of the load regarding the flight direction. Geometry for which the minimal sensitivity is achieved, changes with variable $m_{1,2} / m_{0}$ in such a way that prevalently $d$ changes while the vertical separation $h$ almost does not change. Thus, in that interval of values of $m_{1,2} / m_{0}$ the stability of the flight is achieved prevalently by changes in contribution of the UAV 1 to the load carrying. That is another manifestation of the previously stated fact that in the stated region of changes of $m_{1,2} / m_{0}$ one observes gradual rise in contribution of the UAV 1 to load carrying. For heavy UAVs (or for lightweight load), i.e. $m_{1,2} / m_{0}>10$ geometry stabilises around $d<<L$ and $h \approx L$ corresponding to the situation in which both UAVs contribute to load carrying by opposing the load drag force, since it becomes more pronounced for relatively lightweight loads (cf. left graph in Fig. 4).

\section{CONCLUSIONSOF SIMULATED MODEL}

We analytically treated the stationary level flight of the two UAVs that simultaneously transport a load. The analysis focused onto static stability (28) and power and energy factors (27).

Transport by a single UAV is energetically favourable compared to the transport by two UAVs, because
$\left(T_{1}+T_{2}\right) / \tau>1$ for achievable masses, drag forces and other parameters (see e.g. Fig. 7). Yet, for most of the geometries, the power generated by each of the two UAVs is smaller than the power of a UAV that solitary carries a load. In practice, that means smaller probability of malfunctioning and cheaper maintenance. Lesser power per UAV generated means $T_{1,2}<\tau$ for most of the values of $d / L$, as in Figs. 3-5. For example (Fig. 4) $T_{2}=\tau$ for $d / L=$ 1,43 and $T_{1}=\tau$ for $d / L=1,60$. For $10 \%$ larger $d / L=1,73$ (which is maximal value of $d / L$ in case $h / L=1$ ) one has $T_{2}$ $=6,48 \cdot G_{0} \approx 2,9 \cdot \tau$. So, within $90 \%$ of the interval of possible values of $d / L$ one has $T_{1,2}<\tau$.

Sensitivities are minimal in cases when the thrusts negligibly change for small changes in configuration: in Fig. 8 scaled sensitivity minimum 0,95 is achieved for $d / L=0,33$ and $h / L=1,19$. The change in total thrust (left graph in Fig. 7) is less than $1 \%$ for in the interval from $d / L=0,27$ to $d / L=1$. Configurations corresponding to minimal scaled sensitivity are characterised by the minimal effects of competition between the UAVs: $T_{2}=2,23 \cdot G_{0}$ which is close to $\tau=2,24 \cdot G_{0}$, while $T_{1}=0,99 \cdot G_{1}$ in left graph in Figure 4 . Thus there are virtually no parts of $T_{1}$ and $T_{2}$ to be used by one UAV against the other. If the mass of the load is smaller than or equal to the masses of UAVs, then, effectively, back UAV carries most of the load, while the front UAV carries a negligible amount of the load (Fig. 3): for $d / L=0,27$ one has $T_{2}=1,47 \cdot G_{0}$, close to $\tau=1,49 \cdot G_{0}$, while $T_{1}=0,10 \cdot G_{0}$. Thus the front UAV carries around $7 \%$ of the load weight. If the load has negligible mass compared to that of UAVs' then the drag force of the load is to be opposed by UAVs' thrusts and the geometry becomes more symmetrical in the vertical planes. Then UAVs contributions to load carrying become more similar. In particular (Fig. 5), one has $T_{1}=T_{2}=5,91$ 
for $d / L=0,59$. Moreover, $T_{1}$ and $T_{2}$ are within the same order of magnitude within $50 \%$ of available values of $d / L$.

Overall, if the atmosphere is stationary and energy consumption is the most important factor, then the use of a single UAV is preferred in aerial load transport. Contrary to that, advantages of the load carrying by two UAVs becomes important in cases when wind gusts (here implicitly included in the larger drag forces parallel to $x$-axis) or other sudden changes in atmospheric conditions are expected since two UAVs make possible load carrying with a better stability in the way that most of the sudden changes in required thrust are provided by the UAV that contributes less to the overall load carrying. Furthermore, it is a preferred way of transport also in cases in which the load of the UAV is important, because for most of the achievable geometries that load per UAV is smaller when two instead of one UAV carry a load. Detailed analysis reveals that in such cases one UAV carries most of the load while the other contributes as the source of the backup power.

\section{REFERENCES}

[1] Brambilla, M., Ferrante, E., Birattari, M., \& Dorigo, M (2013). Swarm robotics: a review from the swarm engineering perspective. Swarm Intelligence, 7(1), 1-41. https://doi.org/10.1007/s11721-012-0075-2

[2] Qiu, H., Wei, C., Dou, R., \& Zhou, Z. (2015). Fully autonomous flying: from collective motion in bird flocks to unmanned aerial vehicle autonomous swarms. Science China Information Sciences, 58(12), 1-3. https://doi.org/10.1007/s11432-015-5456-x

[3] York, G. \& Pack, D. J. (2011). Ground Target Detection Using Cooperative Unmanned Aerial Systems. Journal of Intelligent \& Robotic Systems, 65(1-4), 473-478. https://doi.org/10.1007/s10846-011-9590-4

[4] Cosic, J., Curkovic, P., Kasac, J., \& Stepanic, J. (2013). Interpreting Development of Unmanned Aerial Vehicles using Systems Thinking. Interdisciplinary Description of Complex Systems, 11(1), 143-152. https://doi.org/10.7906/indecs.11.1.12

[5] Mester, G., Pletl, S., Pajor, G., \& Rudas, I. (1995) Adaptive Control of Robot Manipulators with Fuzzy Supervisor Using Genetic Algorithms. International Conference on Recent Advances in Mechatronics. 661-666.

[6] Cruz, P. J. \& Fierro, R. (2017). Cable-suspended load lifting by a quadrotor UAV: hybrid model, trajectory generation, and control. Autonomous Robots, 41(8), 1629-1643. https://doi.org/10.1007/s10514-017-9632-2

[7] Pásztor, A. (2014). Gathering simulation of real robot swarm Tehnički vjesnik, 21(5), 1073-1080.

[8] Goodarzi, F. A., Lee, D., \& Lee, T. (2015). Geometric control of a quadrotor UAV transporting a payload connected via flexible cable. International Journal of Control, Automation and Systems, 13(6), 1486-1498. https://doi.org/10.1007/s12555-014-0304-0

[9] Magister, T. (2007). The Problem of Mini-Unmanned Aerial Vehicle Non-segregated Flight Operation. PROMET Traffic \& Transportation, 19(6), 381-386.

[10] Cos, C. D., Acosta, J., \& Ollero, A. (2017). Relative-pose optimisation for robust and nonlinear control of unmanned aerial manipulators. 2017 International Conference on Unmanned Aircraft Systems. https://doi.org/10.1109/icuas.2017.7991363

\section{LIST OF USED SYMBOLS}

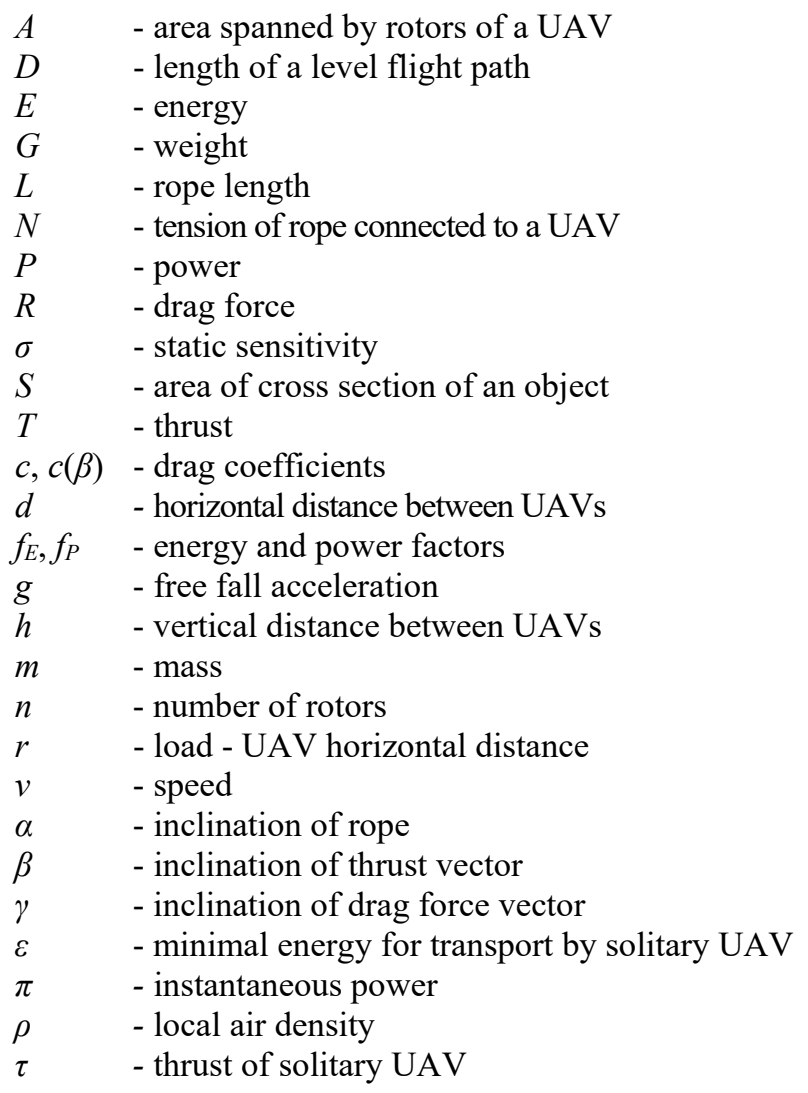

\section{Subscripts}

$0,1,2$ - load, first UAV, second UAV, respectively min - minimal value

$r \quad$ - value for a single rotor of a UAV

$x \quad$ - horizontal projection

$y \quad$ - vertical projection

\section{Contact information:}

\section{Jelena ĆOSIĆ LESIČAR}

Faculty of Mechanical Engineering and Naval Architecture Ivana Lučića 5, 10000 Zagreb, Croatia

jelena.cosic.lesicar@fsb.hr

\section{Dr. sc. Tomislav RADIŠIĆ}

Faculty of Transport and Traffic Science

Vukelićeva 4, 10000 Zagreb, Croatia

tomislav.radisic@fpz.hr

\section{Prof. dr. sc. Tino BUCAK}

Faculty of Transport and Traffic Science

Vukelićeva 4, 10000 Zagreb, Croatia

tino.bucak@fpz.hr

\section{Prof. dr. sc. Josip STEPANIĆ}

Faculty of Mechanical Engineering and Naval Architecture Ivana Lučića 5, 10000 Zagreb, Croatia

josip.j.stepanic@fsb.hr 